\title{
Encouraging Clinicians to Work Effectively with People with Medically Unexplained Symptoms - Is a change in Underlying Attitudes Required?
}

\begin{abstract}
: (250 words)
Medically unexplained symptoms are defined as physical symptoms for which there is no clear diagnosis of organic pathology, including after relevant investigations. Several other terms are also used to describe such symptoms and will be briefly described, although none is ideal.
\end{abstract}

Research will then be summarised, illustrating how patients consulting clinicians in both primary and secondary care often present with symptoms which, whilst undoubtedly distressing for the patient, do not link with any clear organic pathology. This raises difficult issues for clinicians in terms of how much they should investigate and how to manage the patient's problems in a way which will be helpful and mean they will feel their symptoms have been appropriately recognised and addressed. Failure to do this can lead to many negative consequences, including a breakdown in trust between patients and clinicians, overinvestigation or inappropriate treatments, a loss of normal function for the patient and significant costs to the health service and economy.

Despite this, the evidence is that doctors receive very little if any training about how to manage such symptoms at either the undergraduate or postgraduate level. This paper will focus on the attitudes of both junior and more senior doctors across a range of specialities to working with people with unexplained symptoms and how these may affect their management. The implications for clinical practice and recommendations for future training will be discussed and in particular the need to consider the psycho-social as well as the biomedical aspects of patients' presentations from the outset.

\section{Main paper: $(4,902$ words $)$}

In this paper I will be using the term medically unexplained symptoms (MUS) as this is currently the most widely used and probably has a face validity in being comprehensible to the general population as well as clinicians. Many patients have however expressed a dislike of the term and in particular its medical focus and the implication that their symptoms are not explicable or understandable, which can make it seem that they are being disregarded or not taken seriously. Other variations of the term used at times include Unexplained Physical Symptoms (UPS) which has the virtue of simplicity and the longer Medically Unexplained Physical Symptoms (MUPS). An older term is use of the word 'Functional' to describe such symptoms, most often still used with neurological presentations - such as in functional weakness or movement disorders, where the brain is described as being organically normal but to function incorrectly. Interestingly when patient groups are canvassed they appear to prefer this term to MUS, possibly because it gives a sense of providing a diagnostic category with which they can identify [1].

A further potential complication is the overlap between such physical symptoms and how often they may be linked with or be manifestations of psychological or psycho-social problems such as anxiety, depression or the stress associated with difficult life circumstances. There is no doubt that such symptoms or disorders can be very distressing to patients, particularly if not 
managed well, but the frequency of a clear link with a psychiatric mood disorder such as anxiety or depression is open to some debate. Some clinicians appear to suggest that all such presentations are psycho-somatic i.e. have a clear psychological component, but others are less keen to see this as an over-arching factor and research evidence suggests that, whilst coexisting psychological symptoms of anxiety or depression with MUS are common, they are by no means universal $[2,3]$. Patients often resent the emphasis on the psychological without due recognition of the physical nature of many of their symptoms and may feel that they are not being taken seriously or that their symptoms are imagined or 'all in the mind'.

\section{Historical background:}

The need for a new medical model taking into account the psychosocial as well as the biomedical was raised 40 years ago by the psychiatrist George Engel in a landmark paper, raising the paradox of "some people with positive laboratory findings being told they are in need of treatment whilst they are feeling quite well, while others feeling sick are assured that they are well, that is they have no "disease'" [4]. He formulated the concept of the biopsychosocial model, including the patient as well as the illness to encompass both of the above possibilities and allow the patient to feel understood. Despite many references to the importance of employing a biopsychosocial model in many branches of medicine it could be argued there has been relatively little progress made in getting clinicians to actively include this concept in their work. It is also uncertain whether a paper such as that by Engels would nowadays be published in the very high impact 'scientific' journal Science.

Another seminal piece of work was a research study by the US physician Kurt Kroenke published in 1989 [5]. He and his colleagues reviewed the medical records of 1,000 medical patients over a three year period. They were interested in the outcomes for 14 common physical symptoms and found that 38 percent of the patients studied complained of at least one of these symptoms, with diagnostic tests being carried out in two thirds of the cases but an organic/physical cause established in only around $16 \%$ overall and treatment often being ineffective. They suggested that "diagnostic and therapeutic strategies focusing solely on organic causes may be inappropriate for the majority of patients", but this is still the way in which medicine is taught and practised in most Western healthcare settings.

\section{Prevalence and costs of MUS in primary and secondary care in the UK:}

Many research studies over the past twenty years have indicated that unexplained symptoms are very common in both primary and secondary care settings. An important early UK study by Peveler et al. from 1997 [6] following 175 patients identified from appointment lists for general practitioner (GP) clinics indicated that 19\% were identified by their GP as having clinically significant unexplained physical symptoms of at least three months duration, whilst almost twice this number (35\%) had self-identified as having several unexplained physical symptoms on completion of a questionnaire. Around $20 \%$ of the whole sample were identified as having a probable mood disorder, which was higher at around a third in those with unexplained physical symptoms. A recent systematic review of 32 primary care studies from 24 countries involving a total of 70,000 patients has given a higher prevalence rate of 40 to $49 \%$ of patients seen complaining of at least one medically unexplained symptom [7]. Reasons for the very high rates in this study are likely to include the heterogeneity of the studies included, a lower threshold of 
only one unexplained symptom being required for inclusion and a noted variability in quality of the studies, but it does suggest the high frequency with which primary care clinicians are likely to encounter such presentations.

The problem is by no means confined to primary care as revealed in a much quoted study by Nimnuan et al. conducting a retrospective case notes review of patients seen at two London hospitals [8]. 582 (65\%) of the 890 consecutive new patients attending medical out-patient clinics returned completed questionnaires and were included. Of these an average of $52 \%$ of the participants across a range of medical out-patient clinics were described as having medically unexplained symptoms 3 months after their initial appointment - defined as symptoms for which no conventional biomedical explanation could be found after appropriate examination and investigations had been carried out. The highest rate of unexplained symptoms was $66 \%$ in gynaecology clinics, but neurology (62\%), gastroenterology (58\%) and cardiology (53\%) also had a high prevalence, with over half of those seen having no clear organic diagnosis after three months.

These high rates of unexplained symptoms in both primary and secondary care suggest that a significant number of patients are likely to have undergone potentially avoidable referrals and/or invasive investigations which have not led to an organic diagnosis. This is associated with major costs - in terms of difficulties and frustration in the relationship between patients and their clinicians when the biomedical route fails to give an explanation for a person's symptoms, as well as the potential for harm done with invasive tests, the associated anxiety when these fail to give a clear diagnosis and the significant accompanying financial costs. Work by Bermingham et al. has indicated that the costs to the UK economy of medically unexplained symptoms are very high [9], suggesting direct health costs of $£ 3$ billion in the year 2008-9, with wider costs to the economy in terms of absence from work and impaired quality of life of $f 14$ billion.

\section{Undergraduate and postgraduate teaching about MUS:}

All these studies indicate the importance of educating both undergraduate medical students and postgraduate clinicians about the optimum ways to diagnose and manage presentations which do not appear linked with clear organic/physical pathology. However, the evidence is that such teaching is still extremely limited despite a British Medical Journal editorial in 2005 highlighting the paucity of theoretical and practical training in medically unexplained symptoms in most university curricula and GP postgraduate training programmes [10]. In 2011 Howman and colleagues conducted a survey of undergraduate teaching about MUS in UK Medical Schools [11]. They had a 71\% response rate with replies from 22 of the 31 medical schools approached, of whom $86 \%$ (19/22) indicated that there was some teaching about MUS within their undergraduate curriculum. The majority only offered a few hours of teaching which was mainly provided within only one department, most commonly psychiatry, although $42 \%$ $(8 / 19)$ provided some teaching across two or three departments. This is an important consideration, since people presenting with MUS can feature in most specialties and medical settings.

A more recent survey of teaching about this topic provided to junior hospital doctors during their two year UK Foundation programme, a time when young doctors are likely to be having 
their first experiences of managing such difficult presentations themselves, suggested a much lower level of formal education about this important topic [12]. Programme Directors representing 155 Foundation School Programmes across England, Wales and Northern Ireland were asked whether any teaching about MUS was provided within their teaching programmes. Notably the topic of medically unexplained symptoms is not included within the Foundation Year curriculum, but programme directors have some discretion as to what they include in their local training programmes. The response rate was relatively low, with around a third 53/155 responding, and in only 6/53 (11\%) of sites was formal teaching about MUS included, although $22 / 53$ (42\%) of the directors thought the subject was likely to be raised informally in case discussions or Balint type clinical supervision groups. It was considered possible but unlikely that many of those not responding would be involved in providing teaching about the subject.

Views of Foundation year doctors about working with patients with MUS:

The same research team have been involved in a series of qualitative studies investigating the views of a range of qualified doctors about their attitudes to working with patients with MUS and how confident they felt in their management. There were many similarities in the findings across a whole range of clinicians, but also some interesting differences and a summary of the results will be presented here. The most junior clinicians were those in their Foundation years' training and, whilst recognising they had seen a significant number of patients with MUS, they had generally received very little formal training in this topic and often felt unclear about what should be their role and responsibility with such cases, which they felt poorly prepared to deal with. 22 junior doctors from three north London hospitals were interviewed, working in a variety of settings including general practice, accident \& emergency, psychiatry, gastroenterology and care of the elderly [13].

"I felt a bit daunted by the case... and I guess I didn't really feel very prepared to meet people like that, because it's not something that we focus on very much in medical school." (P05, Female, FY1)

"There's so much to learn about stuff that actually you can see and prove goes wrong, and how to treat that, so when you come across something that doesn't fit that dogma you just think well, it's not my job and it's not really my business to be involved in it..." (P23, Male, FY1)

These junior doctors often gave frank descriptions of how seeing such patients without the skills to appropriately deal with them could lead to a sense of nihilism, anxiety and frustration.

"They're frustrating, because there's nothing you can do to help them". (P16, Female, FY2)

"That's the thing I find about medically unexplained symptoms, I've got no answer and I often feel very powerless. I've got nothing to offer you, and I just don't know what to do". (P18, Male, FY1)

They also had difficulty addressing possible psychological morbidity 
"She said the classic line, 'are you telling me it's all in my head doctor?" And whilst we thought it might be something to do with that, it was a really difficult subject to broach with her". (P12, Male, FY1).

Many of these junior doctors were aware that seeing such patients might lead to them being over-investigated and that this might be as much to protect the doctor as for the patient's benefit.

"I think it's all too easy when somebody like that comes in just to do the investigation(s) so you can get rid of them a bit quicker. It takes a lot longer to try and talk to them about it". (P15, Male, FY2)

"There is a lingering fear of missing something or not diagnosing something. Wanting to avoid litigation can sometimes be a driving factor pushing you towards doing lots of investigations". (P07, Male, FY2)

An unexpected finding in this research was the potentially adverse impact of the attitudes of their senior clinicians, who were often experienced as being negative or dismissive towards such patients. Given that such role modelling was the main education which these junior doctors appeared to receive in managing patients with MUS this is of some concern. Several foundation year doctors said that the 'medical culture' meant they felt unable to take more time with such patients or focus on the psycho-social aspects of their presentation.

"Some people [seniors] would sort of scoff at it, and you know, just be derogatory about it". (P17, Female, FY1)

"It's not always what they say, it's the way they say it? The tone of voice, the facial expressions... the nonverbal communication gave me the impression they thought it was a bit of a waste of time, like there wasn't anything wrong with her, and we should try get her discharged ASAP". $\quad$ (P04, Female, FY2)

Views of GP trainees about working with patients with MUS:

A parallel study with GP registrars - young postgraduate doctors involved in a three year training programme for general practice revealed many similar findings, but also some differences [14]. These were doctors who were further along their training path and generally in a position of holding more responsibility for the management decisions they made about the patients they saw, but who were still relatively closely supervised by senior clinicians in their hospital and general practice training posts. One of the significant differences between the experience of Foundation year doctors and the GP trainees was that there were more opportunities for an ongoing relationship with their patients within the general practice component of the GP training.

The GP registrars also often found consultations with patients with MUS challenging and described how they could provoke anxiety, frustration, a sense of feeling overwhelmed and at times anger. They appeared more prepared to express negative attitudes to such patients than the Foundation year doctors. 
"I could find myself getting really agitated with him and getting cross as well ... I've got lots of other things to do. I don't have time for this." (ID 2)

These junior doctors generally appeared much more confident with patients who fitted into the biomedical paradigm. The idea of the body as a machine that can be 'fixed' was used several times, and they seemed much more comfortable when they were able to find a problem, fix it and see an improvement.

"There is something quite nice about being able to say, 'Oh you've got epigastric pain, yes you're $H$ pylori positive, I can do something about it. 'You kind of want to fix it don't you?" (ID 13)

This h. pylori example is a very interesting one, as gastric symptoms suggestive of inflammation or an ulcer were previously put down to stress and doctors often found this hard to manage. When the $h$. pylori bacterium was identified, along with a treatment, many doctors breathed a sigh of relief. This also feeds into the on-going speculation as to whether there may be physical causes for other symptoms currently ascribed to stress.

Most of the GP trainees wanted to be as clear as possible that they were not missing a (physical) diagnosis and struggled to tolerate uncertainty.

"I think once I've reassured myself that it's something that doesn't require further investigation, that it's not something very serious, then I ... put on a different hat with a different lot of skills and manage that patient quite differently, but I would need that reassurance to make sure I'm not missing something."

(ID 14)

This was clearly a conscientious doctor who wanted to do well by the patient. However there is a danger in just pursuing the line of physical investigations until they all come back negative and only then focusing on the possibility of MUS, as patients may find this hard to understand. It is better, if the clinical picture is unclear, to keep both possibilities in mind from the beginning - i.e. using a bio-psycho-social approach - but this is not often formally taught.

The GP trainees were often aware that they might be ordering investigations in such cases in order to protect themselves, but several also felt they had learnt to over-investigate during their hospital jobs.

"One of my professors would [say] 'we'll do a CT anyway because at least then we've done everything and there's nothing more to do. "' (ID 8)

This appears to show little consideration of the potential impact on the patient - e.g. increased radiation, the possibility of anomalous findings, the patient thinking 'there must be something wrong' if an expensive test has been ordered even if the findings are negative.

There were a few GP trainees who appeared to practice in a different way and didn't necessarily focus on primarily excluding physical causes for the patient's symptoms if things were unclear, describing an approach which focused on eliciting the patient's concerns and any 
accompanying psychological factors from an early stage and using these to inform their history taking and management.

"Really trying to pay attention to the psychological side of things early on ... ask them about what else is going on in their life." (ID 4)

There were however also concerns raised by several trainees who felt that following up on psychological difficulties by referring to a mental health specialist might damage the doctor/patient relationship.

"They think they're completely normal and they present with something physical and you say 'Well actually I'm going to send you to the Psychiatrist' ... then that's not going to be great for your relationship." (ID 11)

Views of hospital physicians about working with patients with MUS:

The last set of qualitative interviews was with hospital physicians. The medical specialties chosen were the four with the highest rate of unexplained symptoms at 3 month out-patient follow-up as per the findings of Nimnuan et al. already mentioned and twenty consultants and trainee physicians from three London hospitals were interviewed [15]. Several of the more general findings with regards to the frustration often aroused by these patients and concerns about the potential for over-investigation were similar to the previous studies described, but there were also some specific findings and some notable differences between the junior trainees and more senior consultants.

Some of the clinicians, both junior and senior, felt their role as a specialist was to exclude causes for a patient's symptoms only relating to their own specialty, and not necessarily to go beyond that.

"I see my main function in the heart clinic as reassuring that it isn't coming from their heart and that it isn't something life threatening, rather than telling them exactly what it is".

[Consultant Cardiologist 2]

The danger of such an approach is that it doesn't seem to really consider the patient's perspective - they may well be left wondering what has caused their symptoms and may represent to a different department or specialty.

There was a greater variability in the types of explanation given by these physicians to account for patients' symptoms than in the other two sets of interviews, possibly because they were generally more experienced clinicians as the time spent in hospital training posts is longer at 5 to 7 years, or because holding more specialised posts allowed them to refine the explanations they were able to give.

There were three main types of explanations identified. The first could be described as 'normalising' or giving 'reassurance' that no serious pathology had been found. Many told patients directly that the cause for their symptoms was unknown, some mentioning the 
current inadequacies of medicine to provide an explanation. This approach was particularly taken by hospital trainees. It was also identified in the interviews with the Foundation doctors and many of the GP trainees.

"I'm sure you have that symptom but we can't explain it. What we do know is it's not something worrying or dangerous or life threatening...And it's something that I think we can manage conservatively". [Rheumatology Registrar 7]

A second type of explanation could be described as being physiological or mechanistic and these were used by both junior trainees and consultants as well as some of the GP trainees. The aim was to put the patient's symptoms into a context which they could understand, for example linking their physical symptoms with stress in their lives.

"Most people have a form of stress in their lives and ... if you explore that they can sometimes see ... that the stress from that makes them tense and then you can say that sometimes the tension in your muscles is what's making you feel tired." (GP trainee ID 6)

A third more sophisticated type of explanation could be seen as not just giving an explanation of the patient's symptoms, but also aiming to promoting functional recovery and help patients to achieve control over their symptoms. The evidence is that empowering explanations which make sense to both the patient and clinician are likely to be the most effective.

"...we've looked at the nerves and the electrics and all the messages are getting through. ... and I always couch it in a positive way... We have to find a way in order to get you to make your leg work again." [Neurology Registrar 4]

A distinction amongst the hospital physicians was that several of the more experienced consultants clearly felt more positive about patients who they saw with unexplained physical symptoms and found them interesting and potentially rewarding to treat.

"I like them. I think it's a challenge actually...managing them over quite a long period of time you can, you feel as if you're achieving something ...Looking at their psycho-social issues, so their background, where they come from, what their health beliefs are, what their expectations are and what they expect to happen". [Consultant Rheumatologist 3]

However, being able to provide continuity of care is often not possible within the hospital systems in a hard pressed NHS and the consultants probably had more control over their schedules than many of their trainees who were unable to achieve this.

...the difficulty being a registrar in the NHS is... you rotate through various clinics, so I will not have a longstanding relationship with this patient, which is extremely unfortunate because I can't take it any further. [Neurology Registrar 2] 
All the junior doctors and trainees interviewed across these three studies expressed a desire for proper training in the topic of medically unexplained symptoms and their management and were often aware of the limitations of any training they had received with its emphasis on the bio-medical and learning by trial and error, which are likely to make it a lottery for patients on the receiving end.

"Given that MUS is very common, and actually takes up a heck a lot of resources, I think it's a good idea [to provide training]." (P18, Female, Foundation Year 2).

"In medical school you're taught that patients have things wrong with them and there's always a medical cause for it and you've got to try and find it." (GP trainee ID 4)

"You develop it over time through positive and negative role models, what to do and what not to do." [Neurology Registrar 2]

\section{Discussion and implications for clinical practice:}

It is clear from the background literature and the findings from the qualitative studies described that unexplained physical symptoms are very prevalent across a variety of primary and secondary care settings and widely recognised as a regular part of their workload by both junior and more senior doctors. It is also apparent that many if not most clinicians have had very little training at an undergraduate or postgraduate level in how to best work with such patients. Common themes from the qualitative interviews and background literature were that many clinicians found people presenting with symptoms for which there was no clear diagnosis difficult to work with, often finding them frustrating and anxiety provoking and feeling unprepared for what might be seen as non-medical problems. This is likely to lead to dysfunctional consultations and dissatisfied patients who don't feel that their needs have been addressed and who may re-present in other settings. Doctors across the whole spectrum interviewed were aware that they might over-investigate or over-refer such patients, often for the clinician's rather than the patient's benefit and because of a fear of litigation if they missed a physical diagnosis. Although some of the more senior hospital physicians interviewed expressed an interest in seeing such patients and providing them with consistent care and continuity, there was also evidence from the work with junior doctors that many senior clinicians might hold much more negative views which are likely to impact both on how their junior staff manage such cases and the patients themselves. This is therefore something which needs to be addressed at all levels of the medical profession for any meaningful change to occur.

A finding from the majority of the qualitative data is the reluctance which many of the doctors appeared to have to investigate the psycho-social aspects of patients' histories, focusing very much on the bio-medical. Some very interesting work by a team in Liverpool analysing a series of transcripts of GP consultations with patients with MUS has shown that, whereas these patients often appear to want more emotional support than those with clear organic disease [16], many of the GPs were reluctant to follow-up on any psycho-social cues, which they often disregarded in favour of symptoms which might indicate a physical cause 
meriting investigation [17]. Clearly it is a crucial part of any clinician's role to identify, investigate and treat any significant organic pathology, but ignoring the psycho-social symptoms and history may lead to inaccurate diagnoses and less effective treatments.

\section{Implications for training:}

There was a desire for appropriate training in the identification and management of people with MUS expressed by virtually all the junior doctors interviewed. This was perceived as less of a need by more senior clinicians, but the fact they reported that they had learnt their skills in working with patients with MUS by trial and error during their progression through various hospital posts is hardly an ideal model and potentially leaves patients vulnerable to a variety of approaches, which is likely to be particularly problematic when the diagnosis is unclear.

One of the factors complicating the delivery of suitable training about this topic is the lack of evidence for interventions for medically unexplained symptoms having a direct impact on patients' clinical outcomes such as improved mood, functioning or quality of life. There is however good evidence for specific management techniques in improving clinician skills when communicating with patients with MUS [18] and reducing investigations and healthcare costs [19], so educational interventions focusing on these areas are likely to produce tangible benefits in terms of reduced frustration for both patients and clinicians, increased patient satisfaction and reduced costs.

Giving effective explanations for 'unexplained' symptoms, in a way which make sense to both the patient and the practitioner and help the patient to function better have been shown to be very important [20]. This is an area requiring the development of new skills which could be usefully addressed in training programmes at both undergraduate and postgraduate levels. Highlighting that patients with MUS appear to seek emotional support more than other patients is also important [16], as this may well contribute to the difficulties experienced by many doctors when working with these patients. It is also crucial to emphasise the importance for all doctors of addressing psycho-social factors and patient cues as an inherent part of any clinician's role, with encouragement to consider the possibility of medically unexplained symptoms in parallel with physical diagnoses when cases are atypical and not to have MUS only as a diagnosis of exclusion. The criteria for appropriate referral to other services which may be helpful for patients need to be described - be these psychological therapy services for accompanying mental distress [21] or one of the services being set up for patients with more complex needs [22].

\section{Conclusion:}

Teaching and constructive debate about unexplained symptoms should begin early in a clinician's undergraduate training before attitudes become fixed or polarised, but this needs to be continued into postgraduate training with case discussions and clinical supervision which are relevant to the specialty involved. The reality of uncertainty about a patient's diagnosis in many medical consultations needs to be emphasised and that this does not mean that the doctor is incompetent. Given the perceived negative attitudes of some senior clinicians and the variability of management strategies and approaches currently being 
employed, it is unlikely that introducing training modules only at undergraduate and junior doctor levels would be sufficient to engender the cultural shift required. The importance of providing effective care for all those presenting to medical services within a compassionate biopsychosocial model needs to be emphasised at all levels of the medical hierarchy - if properly engaged with this could lead to more effective consultations, less frustrated clinicians and patients and reduced health care costs.

This is an area of medicine engendering considerable debate and controversy, but of crucial importance in terms of improving patient centred care. This paper has focused on doctors, but the issues raised are likely to also apply in many cases to other healthcare workers.

\section{References:}

[1] Stone J, Wojcik W, Durrance D, Carson A, Lewis S, MacKenzie L, Warlow CP, Sharpe M. (2002) What should we say to patients with symptoms unexplained by disease? The "number needed to offend". British Medical Journal 325, 21 - 28.

[2] Burton C. (2003) Beyond somatisation: a review of the understanding and treatment of medically unexplained physical symptoms (MUPS). British Journal of General Practice 53, $231-9$.

[3] De Waal MWM, Arnold IA, Eekhof JAH, Van Hemert AM. (2004) Somatoform disorders in general practice: Prevalence, functional impairment and comorbidity with anxiety and depressive disorders. British Journal of Psychiatry 184 (6) 470 - 476

[4] Engel GL. (1977) The need for a new medical model: a challenge for biomedicine. Science $196,129-136$.

[5] Kroenke K, Mangelsdorff AD. (1989) Common symptoms in ambulatory care: Incidence, evaluation, therapy, and outcome. American Journal of Medicine 86, Issue 3, $262-266$.

[6] Peveler R, Kilkenny L, Kinmonth A-L. (1997) Medically unexplained physical symptoms in primary care: a comparison of self-report screening questionnaires and clinical opinion. Journal of Psychosomatic Research 42, no. 3, $245-252$.

[7] Haller H, Cramer H, Lauche R, Dobos G. (2015) Somatoform Disorders and Medically Unexplained Symptoms in Primary Care: A Systematic Review and Meta-analysis of Prevalence. Deutsches Ärzteblatt International 112(16), 279 - 287.

[8] Nimnuan C, Hotopf M, Wessely S. (2001) Medically unexplained symptoms: an epidemiological study in seven specialities. Journal of Psychosomatic Research 51, 361 - 7.

[9] Bermingham S, Cohen A, Hague J, Parsonage A. (2010) The cost of somatisation among the working-age population in England for the year 2008-2009. Mental Health in Family Medicine 7(2), 71-84. 
[10] Rosendal M, Olesen F, Fink P. (2005) Management of medically unexplained symptoms. British Medical Journal 330(7481), 4 - 5.

[11] Howman M, Walters K, Rosenthal J, Good M, Buszewicz M. (2012) Teaching about medically unexplained symptoms at medical schools in the United Kingdom. Medical Teacher 34(4), 327 - 9.

[12] Yon K, Habermann S, Rosenthal J, Walters K, Nettleton S, Warner A, Lamahewa K, Buszewicz M (2017) Improving teaching about medically unexplained symptoms for newlyqualified doctors in the UK: findings from a questionnaire survey and expert workshop. BMJ Open In press.

[13] Yon K, Nettleton S, Walters K, Lamahewa K, Buszewicz M. (2015) Junior doctors' experiences of managing patients with medically unexplained symptoms: a qualitative study. BMJ Open http://dx.doi.org/10.1136/bmjopen-2015-009593 (accessed 07.04.2017)

[14] Howman M, Walters K, Rosenthal J, Ajjawi R, Buszewicz M. (2016) "You kind of want to fix it don't you?" Exploring general practice trainees' experiences of managing patients with medically unexplained symptoms, BMC Medical Education DOI: 10.1186/s12909-015-0523-y

[15] Warner A, Walters K, Lamahewa K, Buszewicz M. (2017) How do hospital doctors manage patients with medically unexplained symptoms: a qualitative study of physicians. Journal of the Royal Society of Medicine 110(2), 65 - 72. doi: 10.1177/0141076816686348.

[16] Salmon P, Ring A, Dowrick CF, Humphris G M. (2005) What do general practice patients want when they present medically unexplained symptoms, and why do their doctors feel pressurised? Journal of Psychosomatic Research 59, 255 - 260.

[17] Dowrick CF, Ring A, Humphris GM, Salmon P. (2004) Normalisation of unexplained symptoms by general practitioners: a functional typology. British Journal of General Practice 54(500), $165-70$.

[18] Weiland A, Blankenstein AH, Van Saase JLCM, Van der Molen HT, Jacobs ME, Abels DC, Kose N, Van Dulmen S, Vernhour RM, Arends LR. (2015) Training medical specialists to communicate better with patients with medically unexplained physical symptoms (MUPS). A randomized, controlled trial. PLOS ONE 10:e0138342. doi:10.1371/journal.pone.0138342

[19] Rief W, Martin A, Rauh E, Zech T, Bender A. (2006) Evaluation of general practitioners' training: how to manage patients with unexplained physical symptoms. Psychosomatics 47, $304-311$.

[20] Salmon P, Peters S, Stanley I. (1999) Patients' perceptions of medical explanations for somatisation disorders: qualitative analysis. British Medical Journal 6, 318(7180), $372-376$.

[21] http://www.iapt.nhs.uk/silo/files/medically-unexplained-symptoms-postive-practiceguide-2014.pdf (last accessed 11.04.2017) 
[22] Joint Commissioning Panel for Mental Health. (2017) Guidance for commissioners of services for people with medically unexplained symptoms http://www.jcpmh.info/wpcontent/uploads/icpmh-mus-guide.pdf (last accessed 11.04.2017) 